\title{
One-Stage Clotting Factor Assay
}

National Cancer Institute

\section{Source}

National Cancer Institute. One-Stage Clotting Factor Assay. NCI Thesaurus. Code C159550.

A clotting factor assay where FVIII-deficient plasma is added to test plasma and the APTT reagent, mixed and incubated for 3-5 min. The mixture is then recalcified, and the coagulation time is recorded and compared to a standard curve. 